\title{
Is previous influenza-like illness a potential Trojan horse for COVID-19?
}

\author{
Giancarlo Ceccarelli ${ }^{1,2^{*}}$ (D), Gabriele d'Ettorre ${ }^{3}$, Giuseppe Pietro Innocenti ${ }^{1}$, Claudio M. Mastroianni ${ }^{1,2}$, \\ Massimo Ciccozzi ${ }^{4}$ and Gabriella d'Ettorre ${ }^{1,2}$
}

\section{Dear Editor,}

We read with interest the paper by Verroken et al. underlining that no respiratory virus coinfections were identified in a population of COVID-19 critically ill patients [1]. A recent large cohort study by Nowak et al. confirmed that SARS-CoV-2 and respiratory virus coinfections are rare events observed only in less than $3 \%$, despite the temporal overlap of the two epidemic curves. Interestingly, they observed that $13 \%$ of COVID-19-free patients had isolation from the respiratory secretions of influenza virus, rhinovirus/enterovirus, and coronavirus NL63, in the same period [2]. Although with wide geographical variability, this data is in line with the epidemiology of respiratory viral pathogens in the northern hemisphere during the winter period [3], while deviates from the expected in SARS-CoV-2 infected patients, suggesting a possible viral interference [2].

Otherwise, Italy was affected by the SARS-CoV-2 outbreak since mid-February 2020, immediately after the period in which the incidence of influenza-like illness (ILI) gradually increased until reaching the epidemic peak in the fifth week of 2020, with a level equal to about 13 cases per thousand assisted [4]. The complete epidemiological data from 2019/2020 influenza season are not yet available, but we can assume that the aetiology of ILI was similar to that reported for the 2018/ 2019, when about 8 million of cases were registered in Italy and $31.7 \%$ caused by influenza viruses [4]. In accordance with epidemiological data, we also observed that $63.6 \%$ of 190 COVID-19 patients (admitted to Policlinico Umberto I Hospital of Rome, one of the larger teaching Italian COVID-19 Medical Centers in March 2020) reported in their clinical history a recent ILI (1-3 weeks prior to the appearance of COVID-19-related symptoms). Previous flu vaccination was reported in only $26.3 \%$ of patients. The symptomatology reported for the ILI was mainly characterised by sore throat, cough, runny nose, and conjunctivitis. These findings, although not conclusive, seem to suggest that ILI may represent a risk factor for a subsequent SARS-CoV-2 infection. In confirmation of this, interestingly, a number of ILI-related viral pathogens (i.e. respiratory syncytial virus and influenza virus) have been reported to cause a significant downregulation of ACE2 in the upper and lower respiratory tract, since the early stage after the onset of infection [5]. The consequent reduction of ACE2 activity has been found potentially contributing to severe lung injury and may predispose to a later more severe clinical course of COVID-19 [6]. Moreover, considering that intercurrent viral respiratory infections are a trigger of upper airway mucosal damage and local immune impairment, previous ILI could therefore represent a predisposing factor for subsequent COVID-19 infection. On the basis of these data, influenza vaccine not only has a public health utility permitting to exclude influenza in patients with ILI during the overlapping of the two epidemic curves, but probably also reduces the risk and the severity of COVID-19. Nevertheless, more than $65 \%$ of ILI have a non-flu aetiology; for these reasons, the implementation of behavioural containment measures is needed to reduce the risk of ILI spreads in areas affected by the SARS-CoV-2 outbreak.

\footnotetext{
* Correspondence: giancarlo.ceccarelli@uniroma1.it

${ }^{1}$ Department of Public Health and Infectious Diseases, Sapienza University of Rome, Viale del Policlinico 155, 00161 Rome, Italy

${ }^{2}$ Azienda Ospedaliero-Universitaria Policlinico Umberto I, Rome, Italy

Full list of author information is available at the end of the article
} 


\section{Authors' contributions}

All authors contributed equally. The authors read and approved the final manuscript.

\section{Funding}

None

\section{Availability of data and materials}

On demand to corresponding author

\section{Ethics approval and consent to participate}

Ethical approval was obtained from the Ethics Committee of Policlinico Umberto I (Rome, Italy) [approval no./ID Prot. 109/2020].

\section{Competing interests}

We declare no competing interests.

\section{Author details}

'Department of Public Health and Infectious Diseases, Sapienza University of Rome, Viale del Policlinico 155, 00161 Rome, Italy. ${ }^{2}$ Azienda

Ospedaliero-Universitaria Policlinico Umberto I, Rome, Italy. ${ }^{3}$ Unit of Occupational Prevention and Protection, ASL Lecce, Lecce, Italy. ${ }^{4}$ Department of Medical Statistics and Epidemiology Unit, Campus Bio-Medico University, Rome, Italy.

Received: 10 July 2020 Accepted: 5 August 2020

Published online: 14 August 2020

\section{References}

1. Verroken A, Scohy A, Gérard L, et al. Co-infections in COVID-19 critically ill and antibiotic management: a prospective cohort analysis. Crit Care. 2020; 24:410. https://doi.org/10.1186/s13054-020-03135-7.

2. Nowak MD, Sordillo EM, Gitman MR, Paniz Mondolfi AE. Co-infection in SARS-CoV-2 infected patients: where are influenza virus and rhinovirus/ enterovirus? [published online ahead of print, 2020 Apr 30]. J Med Virol. https://doi.org/10.1002/jmv.25953; (2020). https://doi.org/10.1002/jmv.25953.

3. Visseaux B, Burdet C, Voiriot G, Lescure F-X, Chougar T, Brugière O, et al. Prevalence of respiratory viruses among adults, by season, age, respiratory tract region and type of medical unit in Paris, France, from 2011 to 2016. Plos One. 2017;12(7):e0180888. https://doi.org/10.1371/journal.pone.0180888.

4. Panatto D, Lai PL, Mosca S, et al. Influenza vaccination in Italian healthcare workers (2018-2019 season): strengths and weaknesses. Results of a cohort study in two large Italian hospitals. Vaccines (Basel). 2020;8(1):119. https:// doi.org/10.3390/vaccines8010119.

5. Gu H, Xie Z, Li T, et al. Angiotensin-converting enzyme 2 inhibits lung injury induced by respiratory syncytial virus. Sci Rep. 2016;6:19840. https://doi.org/ 10.1038/srep19840.

6. Zhang H, Penninger JM, Li Y, Zhong N, Slutsky AS. Angiotensin-converting enzyme 2 (ACE2) as a SARS-CoV-2 receptor: molecular mechanisms and potential therapeutic target. Intensive Care Med. 2020;46(4):586-90. https:// doi.org/10.1007/s00134-020-05985-9.

\section{Publisher's Note}

Springer Nature remains neutral with regard to jurisdictional claims in published maps and institutional affiliations. 